\title{
AN INTEGRATED PEDESTRIAN BEHAVIOR MODEL BASED ON EXTENDED DECISION FIELD THEORY AND SOCIAL FORCE MODEL
}

\author{
Hui Xi \\ Young-Jun Son \\ The University of Arizona \\ Systems and Industrial Engineering \\ 1127 E. James E. Rogers Way \\ Tucson, AZ 85721, USA
}

\author{
Seungho Lee \\ Samsung Electronics Co. Ltd. \\ Mechatronics and Manufacturing \\ Technology Center \\ Suwon-City, REPUBLIC OF KOREA
}

\begin{abstract}
A novel pedestrian behavior model is proposed, which integrates 1) Extended Decision Field Theory (EDFT) for tactical level human decision-making, 2) Social Force model (SFM) to represent physical interactions and congestions among the people and the environment, and 3) dynamic planning algorithm involving AND/OR graphs. Furthermore, the Social Force model is enhanced with the vision of each individual, and both individual behaviors as well as group behaviors are considered. The proposed model is illustrated and demonstrated with a shopping mall scenario. Literature survey and observations have been conducted at the mall for data collection and partial validation of the proposed model. The constructed simulation using AnyLogic ${ }^{\circledR}$ software was utilized to conduct several experiments on performance of the mall and scalability of the proposed model.
\end{abstract}

\section{INTRODUCTION}

Human crowd dynamics is an essential factor in designing facilities involving a large crowd considering both emergency conditions (Helbing, Buzna, and Johansson 2005) as well as normal conditions (Parisi, Gilman and Moldovan 2009). Over the past decade, several models have been developed to analyze the underlying mechanism of large-scale crowd behaviors. Xia, Wong and Shu (2009) have classified those models into two major categories: 1) macroscopic models focusing on extremely large crowds whose crowd behaviors are represented via a continuous flow as a whole (Gaskell and Benewick 1987; Xia, Wong and Shu 2009) and 2) microscopic models for studying relatively small crowds whose behaviors emerge from interactions among individuals. As macroscopic models focus on the continuous flow of crowd as opposed to highly variant, individualized behaviors, they have been mostly applied to the crowd behaviors under competitive situations, where panicking individuals are usually driven by their instincts (Helbing, Farkas and Vicsek. 2000). On the other hand, microscopic models pay more attention to individual differences. As our interest in this work is on pedestrian behaviors in a shopping mall, we will focus on the microscopic models.

The social force model (SFM) introduced by Helbing, Farkas and Vicsek (2000) is a widely used microscopic model for various applications (Helbing, Buzna, Johansson et al. 2005; Moussaïd, Helbing, Garnier et al. 2009). While extensive research has been performed to enhance the original social force model with various other aspects, limited research is available in the literature for integrating the human decision-making aspect with the social force models. This has motivated our research. More specifically, the goal of this research is to develop a crowd behavior model, which integrates 1) tactical level human decision-making, 2) operational-level congestions among the people, and 3) detailed level perceptions 


\section{$X i$, Lee and Son}

(e.g. vision) of individuals. In particular, in the proposed crowd behavior model, decisions on selecting one from alternatives (e.g. destinations and movement directions) are made based on Extended Decision Field Theory (EDFT; Lee, Son and Jin 2008), and the physical interactions are represented by the social force model enhanced with the vision of each person (ESFM, which is proposed in this research). In addition, pedestrian group behaviors as well as their communications are also explicitly considered in this work. The proposed model is illustrated and demonstrated with a shopping mall scenario as it provides us with various environmental conditions (e.g. different kind of shops, obstacles, promotions on the shops) and demographic variations (e.g. gender, age, preference, schedule, and grouping). In particular, the scenario has been built based on the shopping corridor of Tucson Mall. To this end, we have developed a simulation model of the considered shopping mall using AnyLogic $($ software, where each individual in the simulation executes 1) EDFT (see Section 2.2), 2) ESFM (see Section 2.1), and 3) dynamic shopping planning (see Section 3.4). Using the constructed crowd simulation model, several experiments have been conducted for various purposes (see Section 4).

\section{PROPOSED INTEGRATED PEDESTRIAN BEHAVIOR MODEL}

The proposed pedestrian behavior model is based on integration of extended Decision-Field-Theory and extended Social Force Model for dynamic congestions among the shoppers and the environment. Figure 1 depicts components of the model and the logical sequence. Each of them is discussed in detail below.

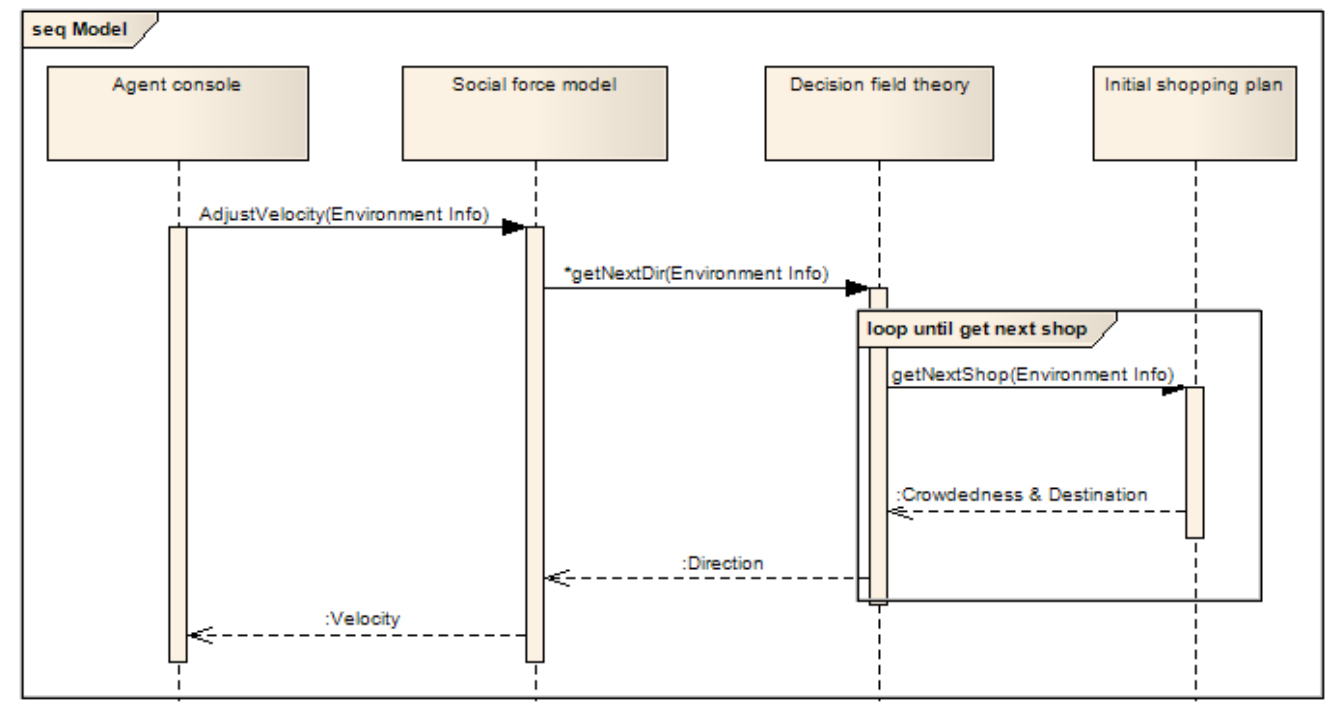

Figure 1: Sequence diagram of components of the model

\subsection{Extended Social Force Model (ESFM)}

Helbing, Farkas and Vicsek (2000) has proposed a Social Force Model, where motives and impacts to a crowd of a pedestrian are represented by a combination of physical and psychological forces (which are translated into the acceleration equation). Equation (1) depicts the formulation of changing velocity at time $t$, where a pedestrian's behavior is determined by his/her desired speed $v_{i}^{0}(t)$ and direction $e_{i}^{0}(t)$ as well as interactions with other individuals and obstacles.

$$
m_{i} \frac{d \boldsymbol{v}_{i}}{d t}=m_{i} \frac{v_{i}^{0}(t) \boldsymbol{e}_{i}^{0}(t)-\boldsymbol{v}_{i}(t)}{\tau_{i}}+\sum_{j(\neq i)} \boldsymbol{f}_{i j}+\sum_{W} \boldsymbol{f}_{i W}
$$




$$
\boldsymbol{f}_{i j}=\boldsymbol{f}_{i j}^{p s y}+\boldsymbol{f}_{i j}^{p h y}, \quad \boldsymbol{f}_{i j}^{p s y}=A_{i} \exp \left(\frac{r_{i j}-d_{i j}}{B_{i}}\right) \boldsymbol{n}_{i j}, \quad \boldsymbol{f}_{i j}^{p h y}=\operatorname{kg}\left(r_{i j}-d_{i j}\right) \boldsymbol{n}_{i j}+\kappa g\left(r_{i j}-d_{i j}\right) \Delta v_{j i}^{t} \boldsymbol{t}_{i j}
$$

The first term on the right side in equation (1) represents the impact from the pedestrian's selfconsciousness while the other two illustrate interaction forces from other pedestrians and the walls, respectively. The interaction force consists of a psychological force resulting from distance between each other, and a physical force inspired by counteracting body compression and sliding friction. The total force exerted by pedestrian $j$ to pedestrian $i$ is calculated as equation (2).

While the original Social Force Model (Helbing, Farkas and Vicsek 2000) has been extensively applied to pedestrian behavior modeling, there exist two improvement opportunities. First, the original SFM computes a force impact between every pair of agents in the environment. In other words, there will be a force even between agents who are significantly far away from each other. Second, the social force between agents is always positive implying that all agents are psychologically against each other, which is not the case for friends or family members. To address these two problems, we extended the original SFM. Details of each modification will be discussed in Sections 2.2 and 2.3.

\subsection{Connection Range Impact on Social Force Model}

In our ESFM, we define a connection range, $C R$, for each agent in the environment. Before applying the force (see first equation in (2)) between two agents, the ESFM will evaluate the distance, $d_{i j}$, between them first and compare it with the connection range. Only if $d_{i j}<C R$, these two agents are connected and have a force affecting their movement (see first equation in (3)). However, there is an exception for the group members, which will be discussed in Section 4.1.

$$
d_{i j}=\left\{\begin{array}{l}
>C R \text { agent } i \text { and } j \text { are not connected } \\
<=C R \text { agent } i \text { and } j \text { are not connected }
\end{array}, \quad I_{i j}=\left\{\begin{array}{l}
1, \quad i \text { and } j \text { are group members } \\
-1, \text { otherwise }
\end{array}\right.\right.
$$

\subsubsection{Psychological Attraction between Group Members}

Pedestrian populations in the shopping mall (case study in this research) can be categorized into two types: individual shoppers and group shoppers (see Section 3 for more details about the considered scenario). Among individual shoppers or shoppers from different groups, a psychological force in the original SFC is applicable to keep a comfort distance between them. However, for shoppers belonging to the same group, the psychological force given by first equation in (2) will prohibit them from staying close each other. Therefore, we propose a modification into the psychological force for the members in a same group (see Section 4.1 for more details), where an intimate factor $I_{i j}$ (see second equation in (3)) is multiplied with the psychological force. The main idea is that a positive psychological force is applicable for the people belong to different groups while a negative psychological force is applicable for the people belong to the same group.

\subsubsection{Pedestrians' Reactions according to Their Visions}

In the original SFM, obstacles located at the same distance (without considering the concept of vision or sight) from a pedestrian enforce equal psychological force on him/her. In a real shopping environment, however, people usually pay more attention to the objects within their vision than to those out of their sight. To resolve this problem, we incorporate this concept of vision by defining a visible area for each agent. From the view of an agent, only neighbors in his visible area could affect his movement with the psychological force. Neighbors on his back (out of vision) may provide influence only via a physical force (e.g. contact). In our proposed model, a visible area (range) is defined with a half circle in front of 


\section{$X i$, Lee and Son}

each pedestrian ( $\left( \pm 90^{\circ}\right.$ angle from the pedestrian's current moving direction) (see Figure 2(a)). The vision formula is given in first equation in (4), where $\varphi_{i j}(t)$ is the angle between direction $\boldsymbol{e}_{i}(t)$ and normalized vector $\boldsymbol{n}_{i j}(t)$. With $\varphi_{i j}(t)>90^{\circ}, \cos \left(\varphi_{i j}(t)\right)(<0)$ is rounded up to 0 , while $\varphi_{i j}<90^{\circ}$ will round $\cos \left(\varphi_{i j}(t)\right)(\geq 0)$ up to 1 . Based on this visible area, a modified social force exerted from pedestrian $j$ on pedestrian $i$ is given in second equation in (4).

$$
\cos \left(\varphi_{i j}(t)\right)=\frac{\boldsymbol{n}_{i j}(t) \boldsymbol{e}_{i}(t)}{\left\|\boldsymbol{n}_{i j}(t)\right\| \boldsymbol{e}_{i}(t) \|}, \quad \boldsymbol{f}_{i j}^{p s y}=A_{i} \exp \left(\frac{r_{i j}-d_{i j}}{B_{i}}\right) \boldsymbol{n}_{i j} I_{i j}\left[\cos \left(\varphi_{i j}(t)\right)\right]
$$

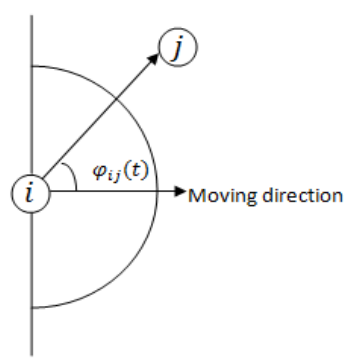

(a)

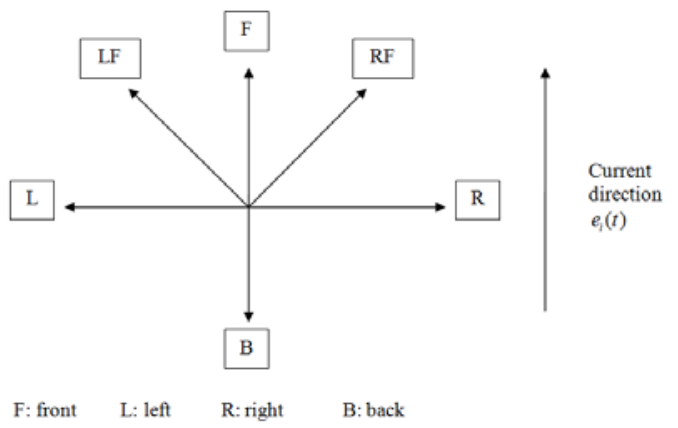

(b)

Figure 2: Visible area for each agent and potential directions to choose for each decision making

\subsubsection{Incorporating EDFT into the Pedestrian Model}

It is generally agreed that decision making about walking trips takes place simultaneously at multiple levels: 1) decision about basic strategy of the trip, 2) route choice, and 3) local spatial behavior considering velocity, trajectory, stops, and attention direction (Zacharias, Bernhardt and Montigny 2005). In this work, it is assumed that a basic strategy (which shops to stop by) and a route choice (in what sequence) are fixed for each individual. Therefore, pedestrian's decisions of our interest are focused on changing their movement directions. Since pedestrians adjust their actual direction from time to time due to the interaction force, the EDFT is employed in this work to mimic this dynamic human decision deliberation process.

Decision Field Theory (DFT) is a psychology-based model and has been widely used for mimicking human deliberation process in making decisions under uncertainty (Busemeyer and Diederich 2002; Busemeyer and Townsend 1993). Lee, Son and Jin (2008) extended the original DFT to cope with a dynamically changing environment. In this work, we integrate EDFT into our proposed pedestrian model to better mimic pedestrians' deliberation on direction changes. Our EDFT is able to model 1) the change of evaluation on the options and 2) the change of human attention along with the dynamically changing environment. The formulation of EDFT is given in equation (5).

$$
P(t+h)=S P(t)+C M(t+h) W(t+h)
$$

Definitions of the main elements of EDFT are explained below:

- $M(t)$ is the value matrix ( $n \times m$ matrix, where each of $n$ options has $m$ attributes) representing the subjective perceptions of a decision-maker by $M(i, j)$. In our case (choosing a direction), a pedestrians have six options (see Figure 2(b)), and each direction corresponds with two attributes (crowd density and destination) that affect their choice. If the next destination is within direction $i$, the entry value $\operatorname{Des}_{i}(t)$ is 0.5 ; otherwise, it is 0.1 . To decide whether a destination is within a particular direction, we use $\boldsymbol{n}_{\mathrm{DD}}$ to denote a vector from pedestrian $i$ to the destination. If the angle 


\section{$X i$, Lee and Son}

between $\boldsymbol{n}_{\mathrm{iD}}$ and the direction is less than or equal to 22.5 degree, we claim that the destination is in this direction. Thus, the value matrix $M$ has a dynamic representation as shown in equation (6), and its values change whenever underlying conditions change.

- $W(t)$ is a weight vector allocating the portion of human attention to each column $j$ (attribute) of $M$ through $W(j, 1)$, which is the only dynamically changing element in the original DFT. In the shopping mall environment, it is assumed that pedestrians intend to arrive at their destination as soon as possible. However, when the environment is really crowded, they tend to put more weight on the impact of crowd density. Equation (7) depicts $W(t)$ used in the considered shopping mall scenario, where $c d_{i}(t)$ denotes the crowd density.

- $S$ demonstrates the stability of preference to each option by its structure. The diagonal elements of $S$ represent the memory from the previous preference state while off-diagonal elements give the inhibitory interactions among competing options. Here, it is assumed that the same amounts of memory and interaction effects are given to the options: 1) Matrix $S$ is assumed to be symmetric, and 2) diagonal elements of $S$ are assumed to have the same value. Moreover, all eigenvalues $\lambda_{i}$ of $S$ are less than one in magnitude to make the linear system stable $\left(\left|\lambda_{i}\right|<1\right)$. Besides, from Figure 2(b), we can see larger interactions between directions within 45 degree than those in 90 or larger degrees.

- $C$ is the contrast matrix comparing the weighted evaluation of each option, $M W(t)$. In our case, each option is evaluated independently, thus $C$ tends to be $I$ (identity matrix).

Given the aforementioned elements and our six-option scenario, the corresponding DFT formula, as defined in equation (5), is described in equation (8).

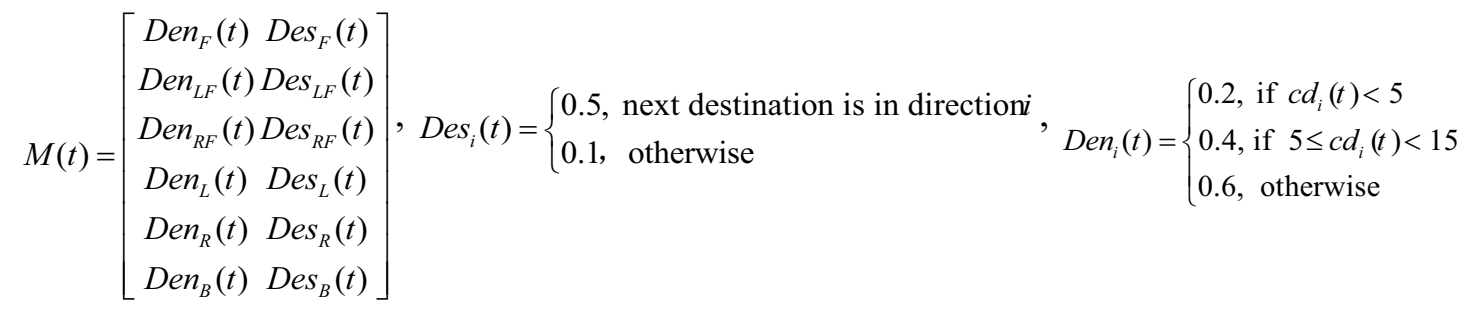

$$
W(1,1)= \begin{cases}{[0.2,0.3]^{T},} & \text { if } c d_{i}(t)<5 \\ {[0.25,0.6]^{T},} & \text { if } 5 \leq c d_{i}(t)<15, \quad W(1,2)=1-W(1,1) \\ {[0.4,0.6]^{T},} & \text { otherwise }\end{cases}
$$

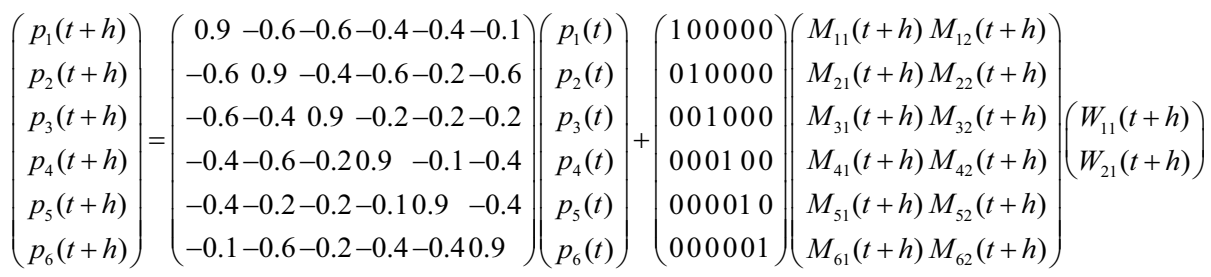

\section{DEVELOPMENT OF AGENT-BASED SIMULATION BASED ON PROPOSED PEDESTRIAN MODEL}

This section describes development of a crowd simulation model for a shopping mall scenario, where behaviors of individual shoppers are based on the proposed, integrated pedestrian behavior model (see Section 2). Agents evaluate the surroundings and try to make optimal decisions so as to achieve their intention. Figure 3 depicts a state chart for shoppers, which contains different states in which shoppers will be 


\section{$X i$, Lee and Son}

in and their transitions. More details about each state and simulation models will be discussed in detail in the following sections.

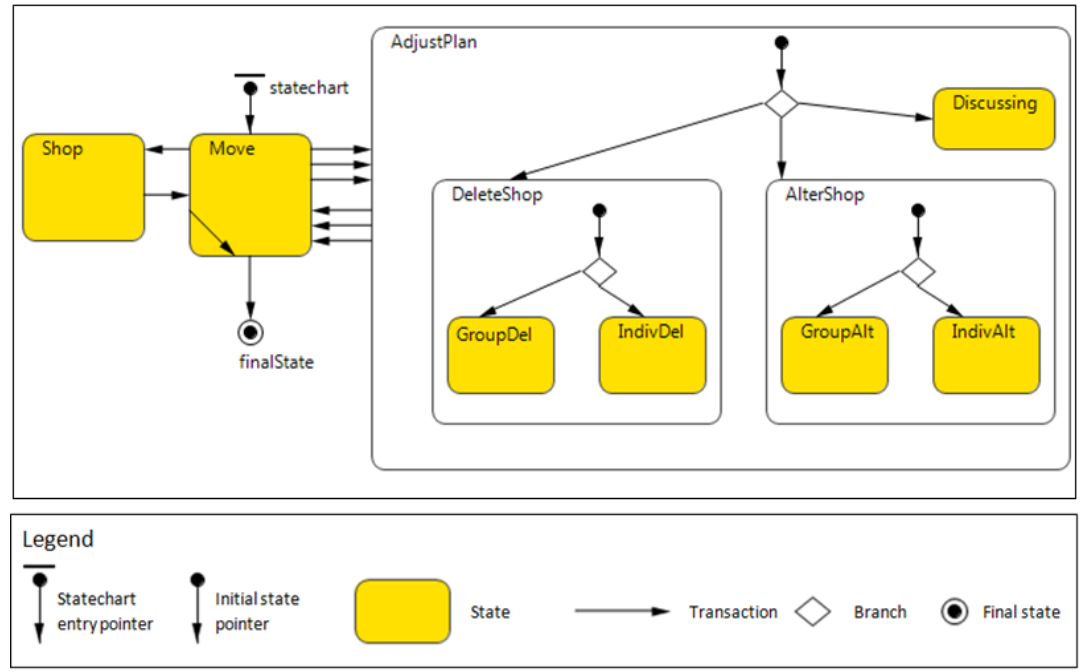

Figure 3: State charts for the shopper's behavior

\subsection{Shopping Mall (Case Study) and Customer Classifications}

A shopping mall scenario has been designed based on the directory of Tucson Mall (the largest shopping mall in Tucson, AZ), and its simulation has been implemented using AnyLogic ${ }^{\circledR} 6.4$ agent-based simulation software. The considered scenario covers eight shops in four different types: three clothing shops, two sports shops, two beauty shops, and one candy shop. Each type of shops has its target customers. Table 1 depicts multiple categories of customers considered in this work. First, customers are tagged with three agent types based on their gender and age: 1) female adult, 2) male adult, and 3) child. In our simulation, agent type is determined based on the discrete, empirical statistical distribution shown in equation (9).

$$
P_{\text {agenttype }}= \begin{cases}{[0,0.5],} & \text { agent type is female adult } \\ (0.5,0.8], & \text { agent type is male adult } \\ (0.8,1.0], & \text { agent type is child }\end{cases}
$$

Table 1: Agent categorizations.

\begin{tabular}{|c|c|c|c|c|}
\hline \multicolumn{2}{|c|}{ Shopping style } & \multicolumn{2}{c|}{ Agent type } \\
\hline \multicolumn{2}{|c|}{ Unplanned } & \multicolumn{2}{|c|}{ Female adult } & Male adult \\
\hline \multirow{2}{*}{ Planned } & Group & Female adult & Male adult & Child \\
\cline { 2 - 5 } & Individual & \multicolumn{2}{|c|}{ Female adult } & \multicolumn{2}{c|}{ Male adult } \\
\hline
\end{tabular}

Second, based on the shopping style, customers are categorized to planned shoppers (people who go to the mall with specific shopping plans) and unplanned shoppers (who do not have a specific shopping plan). Upon arriving at the mall, planned shoppers already have which shops they will visit in mind. Planned shoppers are further partitioned into group shoppers (those who do shopping with friends or family members) and individual shoppers. In this work, it is assumed that all the unplanned shoppers are individual shoppers. By combining agent type with other categorizations (e.g. planned vs. unplanned shoppers; individual vs. group shoppers), we can enhance flexibility of pedestrians' behaviors as well as their adaptability to the environment. 


\section{$X i$, Lee and Son}

\subsection{Algorithm for Movement of Pedestrians}

In this section, algorithm for movement of pedestrian is discussed in detail. Figure 4 depicts a flow chart of the movement algorithm. As discussed in Section 2.2, a desired destination is used as part of input $M$ for the EDFT during the decision deliberation on directions. For the planned shoppers, a potential destination is obtained from their shopping plans. On the other hand, unplanned shoppers normally set the closest shop as their potential destination. Details about evaluation of the destination are illustrated below in Sections 3.3 and 3.4 for unplanned shopper and planned shoppers, respectively. As soon as an agent (shopper) comes up with a desired destination, he/she utilizes EDFT to determine their next moving direction. Then, by calculating physical and social forces based on the surroundings along the moving direction, each agent adjusts its velocity in SFM (see equation (1)).

\subsection{Destination Confirmation Algorithm for Unplanned Shoppers}

Unplanned shoppers do not know in advance which shop they will visit. When they pass by a shop, they will set it as a potential destination if the shop's crowdedness level is below their threshold. Then, they evaluate the shop based on their personal interest and the shop's attraction level by equation (10) which depicts a probability function on whether unplanned shoppers enter a shop or not.

$$
P_{i w}=\alpha I_{i w}+\beta A_{i w}
$$

$I_{i w}$ denotes the interest level of agent $i$ for shop $w$ while $A_{i w}$ describes the attraction level of shop $w$ towards agent $i$. Constants $\alpha$ and $\beta$ are the weight values assigned to the interest level and the attraction level, respectively. Both variables $\left(I_{i w}, A_{i w}\right)$ and constants $(\alpha, \beta)$ range from 0 to 1 . In our model, we give the same weight to $I_{i w}$ and $A_{i w}$ by setting $\alpha=\beta=1$. Therefore, in the normalization step, the value obtained for $P_{i w}$ is divided by 2 in order to obtain its normalized value. If the normalized $P_{i w}$ is above the threshold value 0.7 , agent $i$ will enter shop $w$.

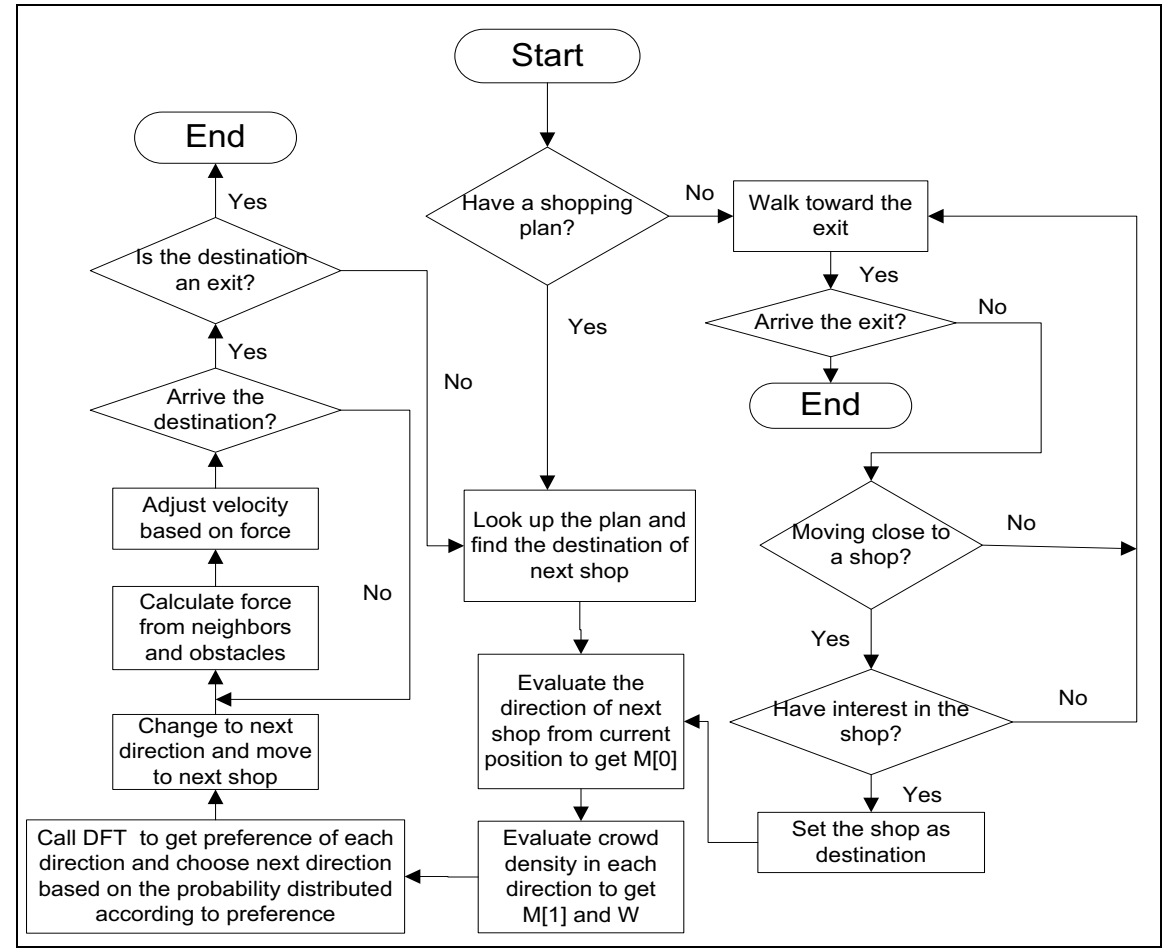

Figure 4: Pedestrian moving algorithm 


\section{$X i$, Lee and Son}

\subsection{Planning Algorithm for Planned Shoppers}

Planned shoppers obtain their potential destinations based on their shopping plan, and evaluate them in the same way that unplanned shoppers do (see Section 3.3). If the potential destination does not meet one of their three criteria (interest level, shop's attraction level, and shop's crowdedness level), planned shoppers will need to decide whether they will skip the shop or move to another shop of the same kind. This decision process is defined as plan adjustment. The following sections discuss design of initial shopping plans and the plan adjustment algorithm for planned shoppers in greater detail.

\subsubsection{Alternative Initial Shopping Plans for Planned Shoppers}

When planned agents arrive at the entrance of the mall, they will be offered with initial shopping plans according to their characteristics (see Table 1). For individual planned shoppers, the plan is designed based on their agent type. For instance, female shoppers may want to visit all three clothes shops and two beauty shops if their schedule permits. Thus their initial shopping plan will include these shops. For a male shopper, a different plan will be designed according to his personal need. Table 2 depicts shopping plans for individual shoppers. Plans in Table 2 contains alternatives in stores to visit (using OR junctions) or in the sequence of stores to visit (using AND junctions).

Based on the observations made at the mall, we have partitioned shopping groups into three types: 1) female groups; 2) mixed-gender groups with both male and female members; and 3) family-with-kid groups. Family groups are mixed-gendered groups, but they will include candy shops on their plan due to kids. For group shoppers, their shopping plans are not based only on one person's interests, but should consider the need of all the members in the group and achieve a balance for the whole group's interests. Taking a mixed-gender group for example, while female members may need to visit more beauty shops and males may need to visit sports shops, the group shopping plan would include both types but only one shop (less than what's preferred by each party) for each type. When group shoppers enter the mall, they will be assigned with a group ID $(0 \sim 8)$, which will indicate the group type that they belong to. Table 3 depicts group types and corresponding initial plans.

Table 2: Shopping plans for individual shoppers.

Table 3: Shopping plans for group shoppers

\begin{tabular}{|c|c|}
\hline Agent Type & Initial Shopping Plan \\
\hline Female Adult & (A) $\quad$ C) $-\mathrm{F}$ (H) \\
\hline Male Adult & (s) (ii)-(aa) (ia)-si) (io) \\
\hline \multicolumn{2}{|c|}{$\begin{array}{l}\text { Legend: } \\
\text { (A)-- } 15 \text { : shop (see Figure } 6 \text { (a) for shop types) } \\
\text { : separate end of OR operation } \\
\text { (9: joint end of OR operation } \\
\text { @: separate end of AND operation } \\
\text { (a): joint end of AND operation }\end{array}$} \\
\hline
\end{tabular}

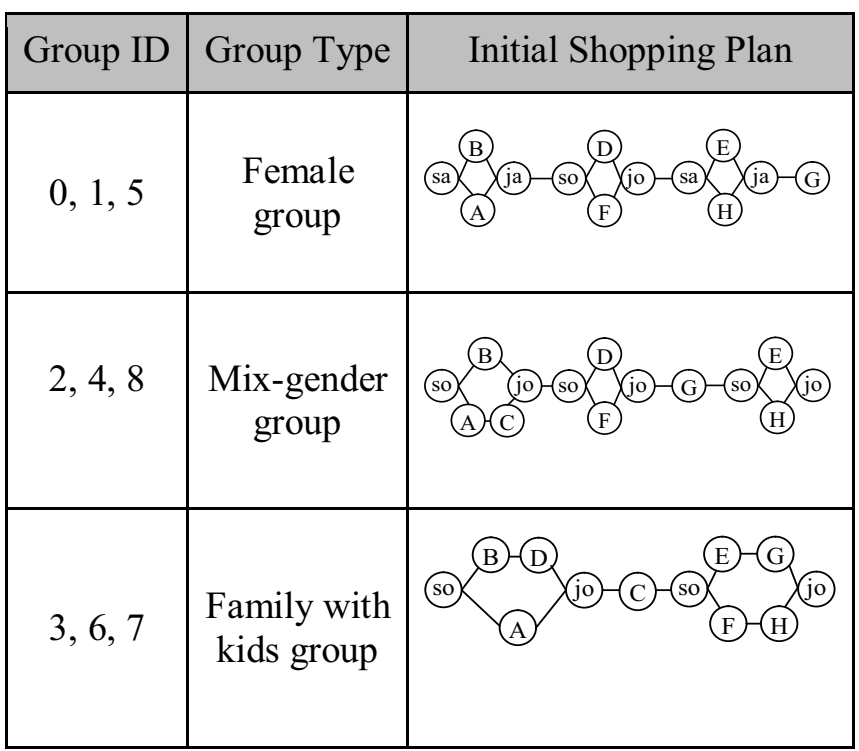




\section{$X i$, Lee and Son}

As shown in Tables 2 and 3, initial shop plans contain alternatives, and one of them will need to be selected based on the current situation. Taking a female adult as an example in Table 2, she needs to visit shop A and shop B upon her arrival, but in any sequence. This selection is based on both her current position and the shop selection probability. If she enters the mall at area 1 in Figure 6(a), the probability of selecting shop B is higher than that of shop A because of its proximity. We use $P_{s w}$ to denote the probability that shop $w$ will be selected by a nearby agent and $\operatorname{Pos}_{k}$ to denote the position of agent. Then the probability of selecting shop A and shop B as the first destination is given by equation (11).

$$
\begin{gathered}
P_{A}=P O S_{2} * P_{s A}+\operatorname{Pos}_{1} *\left(1-P_{s B}\right), \quad P_{B}=P o s_{1} * P_{s B}+P o S_{2} *\left(1-P_{s A}\right) \\
P_{o s_{k}}= \begin{cases}1, & \text { if an agent's entering position in area } i ; \\
0, & \text { otherwise }\end{cases}
\end{gathered}
$$

\subsubsection{Plan Adjustment Planned Shoppers}

As described in Section 3.4, a potential destination of planned shoppers becomes a confirmed one if the shop's crowdedness level is below their threshold. If a considered shop is too crowded, they may want to skip it and go to a next planned one or adjust their plan to visit a different shop of the same kind. For individual shoppers, they evaluate a shop according to equation (10) just as unplanned shoppers do. If $P_{i w}$ (stop by pro) is larger than 0.5 , they will choose to visit a similar shop instead. Otherwise, they will skip the shop and set a next planned shop as the potential destination.

While individual shoppers make decisions on selecting and adjusting their shopping plans on their own mind, members of the same planned group always follow the same plan and proceed together. Detailed design of shopping plans has been already discussed in Section 3.4.1. As shown in the start charts of the proposed model (see Figure 3), if a group member $j$ wants to modify the group plan (e.g. skipping a planned store $w$ ), he/she first sends a "skip" message to all the other group members, then moves his/her state from the "Move" state to the "Adjust plan" state. Upon receiving the message, other group members will enter the "Discussing" state in which they evaluate the message individually with their interests and store the attraction level based on Equation (10). After all the group members come up with a visiting probability $P_{i w}, P_{g w}$ (a group visiting probability) is calculated based on Equation (13), where $g$ denotes a group ID and $n$ denotes the group size.

$$
P_{g w}=\frac{\sum_{i(\neq j)} P_{i w}}{n-1}
$$

Similar to the case with individual shoppers, if $P_{g w}$ is above the threshold value 0.7 , the group accepts member $i$ 's suggestion and deletes shop $w$ from their planned list. Otherwise, all group members including $i$ will still consider shop $w$ as a next destination. Altering stores to be visited follows the same rule.

\section{EXPERIMENTS AND RESULTS}

Using the crowd simulation model constructed based on the proposed pedestrian model and data (survey and observations), we have conducted several experiments for various purposes. The detailed design of each experiment, results, and analyses are described in the following sections.

\subsection{Significance of Consideration of Vision in Social Force Model}

The goal of this experiment is to test significance of consideration of human's vision into the social force model, which is part of the proposed pedestrian behavior model in this work. As mentioned in Section 2, 


\section{$X i$, Lee and Son}

one of the group characteristics is the positive social force among the group members in a same group who always stay close to each other and move together. Even if two group members are out of their connection range (CR, see Section 2.1), there is still a psychological force $f^{p s y}$ between them in order to reduce a distance between them. Once they get closer and are within the connection range, a physical force $f^{p h y}$ begins working to avoid any friction or collision between them. Figure 5 depicts the forces between group members from a group member $i$ 's view. Equation (14) depicts the resultant force function for the group members.

$$
\boldsymbol{f}_{i j}= \begin{cases}\boldsymbol{f}_{i j}^{p s y}+\boldsymbol{f}_{i j}^{p h y}, & d_{i j}<=C R \\ \boldsymbol{f}_{i j}^{p s y}, & d_{i j}>C R\end{cases}
$$

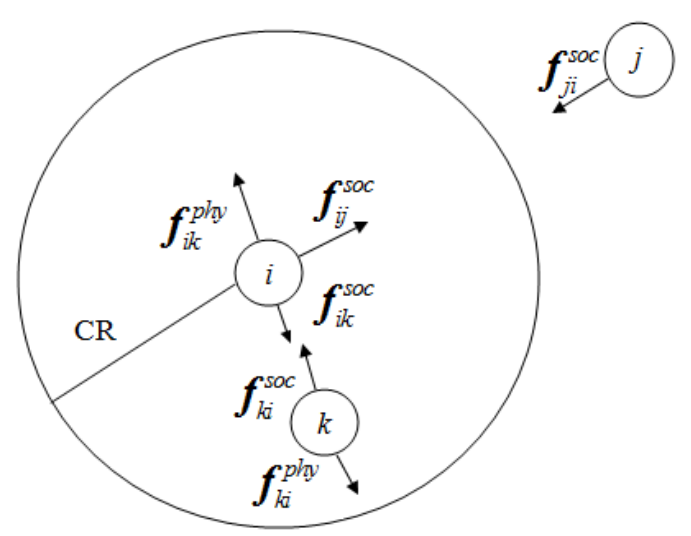

Figure 5: Force execution between group members (from group member $i$ 's view)

By considering the human vision in the SFM, pedestrians will have a psychological force against only those neighbors in front of them which consequently reduces their resistance force and therefore increase their speed. Figure 6(a) depicts a snapshot of the shopping mall simulator that we have developed, where 100 pedestrians are moving along the hallway towards the exit. The average distance to neighboring shoppers and speed of pedestrians are used as the metrics. Figure 6(b) and (c) depicts experimental results, where the above mentioned metrics are compared between models with and without consideration of human's vision. The experimental results reveal that our intuition on faster movement of pedestrians when we consider human's vision is correct. Therefore, consideration of human's vision into the SFM is found to be significant.

\subsection{Impact of Unplanned Shoppers on the Number of Visits to Shops}

As mentioned in Section 3.3, shoppers (planned and unplanned) will evaluate the crowdedness of a shop before entering it. The first equation in (15) depicts the probabilities that planned shoppers and unplanned shoppers will purchase items used in this experiment. The second equation in (15) then depicts the profit of shop $w$, where $m$ denotes the minimum crowdedness threshold of the shopper in the shop. If shops are mostly filled with unplanned shoppers, they may lose the opportunity to attract planned shoppers whose probability of purchasing is higher, reducing the profit of the shop.

$$
\operatorname{Pr}_{i}(\text { purchase })=\left\{\begin{array}{ll}
0.8, & i \text { is a planned shopper } \\
0.5, & i \text { is an unplanned shopper }
\end{array}, \quad \text { ProScore }{ }_{w}=\sum_{i}^{m} \operatorname{Pr}_{i}\right. \text { (purchase) }
$$




\section{$X i$, Lee and Son}

To verify the above intuition, we conducted experiments by increasing the same number of planned shoppers and unplanned shoppers respectively. For sensitive analysis purpose, we started the experiment with 130 shoppers. The results demonstrated that adding 44 unplanned shoppers did not increase the profit score obvious. However, adding the same number of planned shoppers increased the profit score from 125 to 250 . Then, an experiment involving a low density environment has been also conducted with 29 planned shoppers and 39 planned shoppers. By adding 20 more unplanned shoppers and planned shoppers into the mall, respectively, less difference are observed compared with the case with higher density environment. According to our experiments, it has been found that the impact of the number of unplanned shoppers on the profit of the mall is greater when the mall is more crowded (e.g. during holidays or weekend).
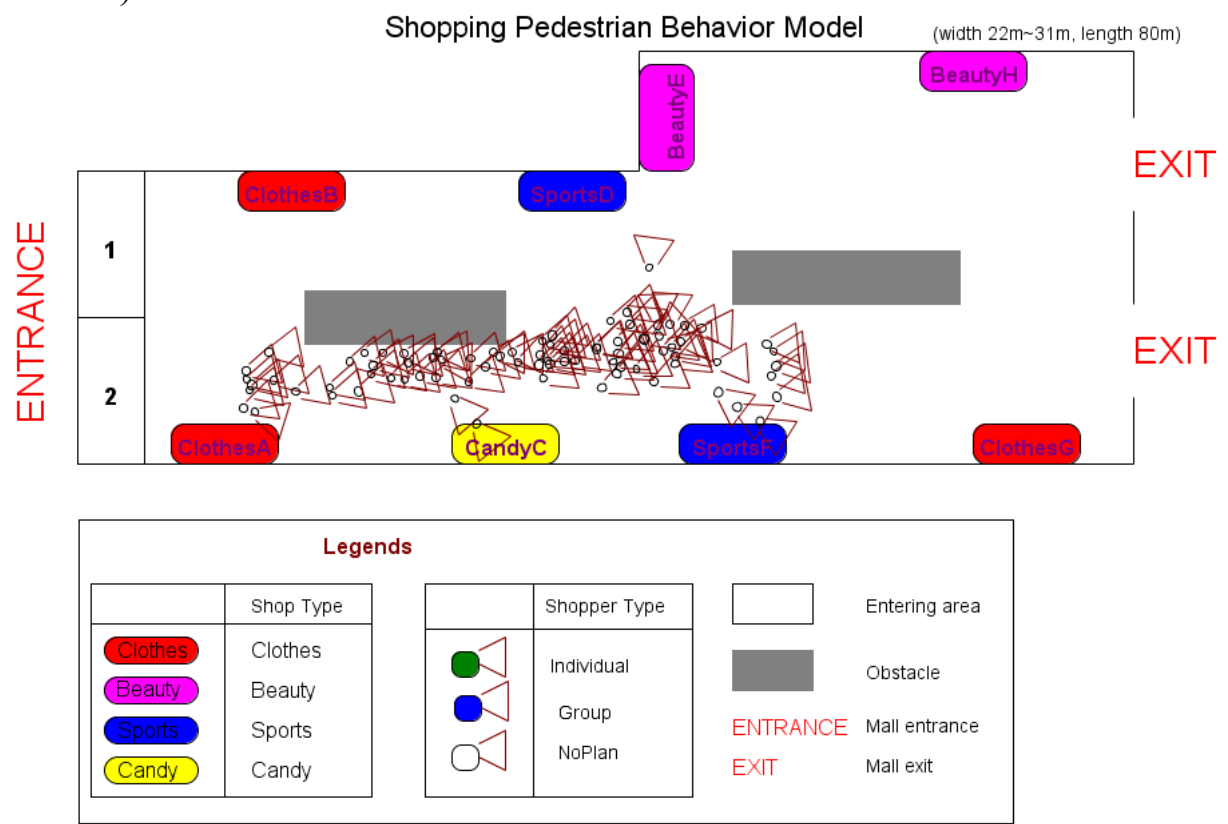

(a) Snapshot of a shopping mall simulation

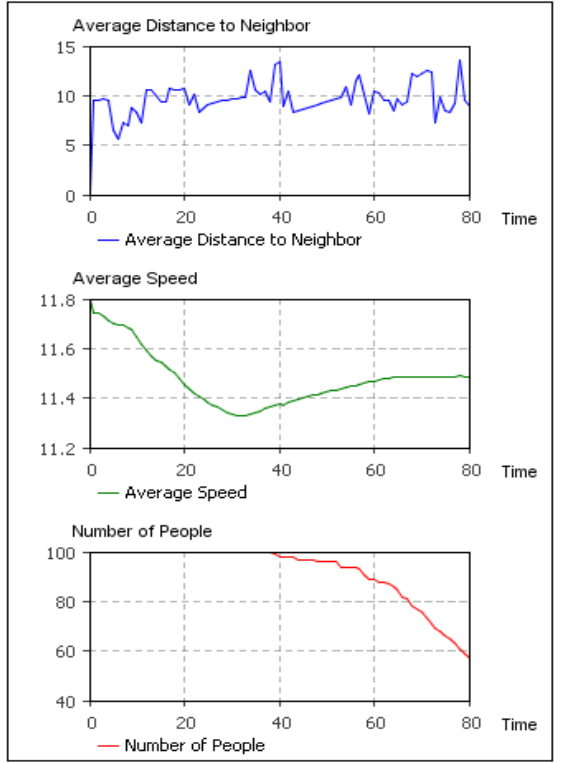

(b) Without vision

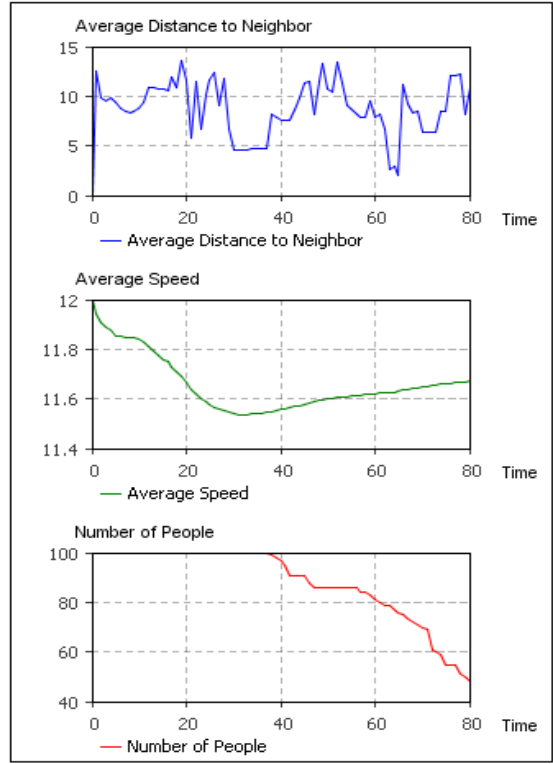

(c) With vision

Figure 6: Comparison results from models with and without consideration of human's vision 


\section{$X i$, Lee and Son}

\subsection{Impact of Group Shopping Behavior on the Profit of Mall}

As mentioned in Section 3.4.2, when a member in a group wants to skip a planned shop or go to an alternative shop, he/she needs to communicate (discuss) with all the other group members first and follow the group's final decision. Therefore, the chance that group shoppers skip or alter a shop is lower than that of individual shoppers. The goal of this experiment is to test our intuition that the shopping mall will gain more profit as the percentage of group shoppers increases. Given the same number of shoppers in the mall (100 shoppers in our case), we examined the results by changing the ratio of group shoppers. It is clearly shown that the profit score significantly increases as the percentage of the group shoppers increases from 0.5 to 0.7 .

\subsection{Arrangement of Stores}

The goal of this experiment is to test the impact of arrangement of stores in the considered shopping mall on their profit score. Two different configurations have been considered: 1) same-type shops are placed far from each other and 2) same-type shops are placed close to each other. The experimental results reveal that the shopping mall gains a higher profit for the second configuration.

\subsection{Scalability and Computational Aspects}

In this research, several efforts have been made to enhance the validity of the crowd simulation model for the considered shopping mall, such as 1) adopting EDFT to mimic decision deliberation of each individual pedestrian , 2) incorporation of explicit group communications, and 3) consideration of human's vision into pedestrian's movement. However, it is expected that these additions will result in longer simulation execution times. The goal of this experiment is to test the scalability of the proposed model. As shown in Figure 7, simulation execution times increase near linearly when the number of agents increases. Therefore, it is believed that our modeling approach is extensible to more complex situations without involving significant increase in the computational time.

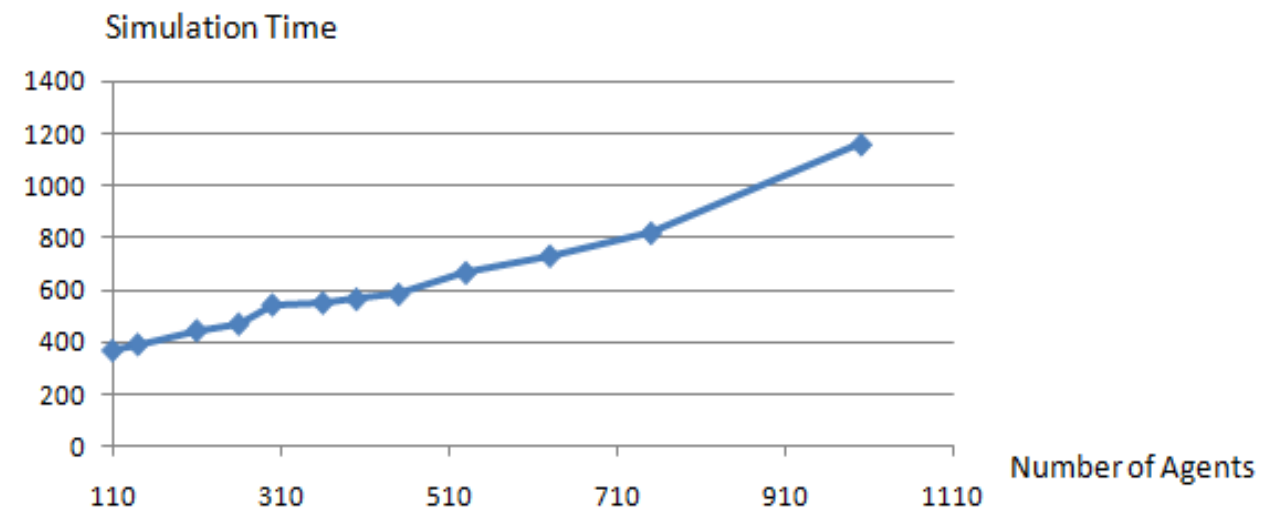

Figure 7: Impact of group behavior on the profit and simulation execution times

\section{CONCLUSION}

The integrated pedestrian model proposed in this paper has allowed us to develop a more realistic simulation of pedestrian behaviors at a shopping mall. In particular, consideration of vision of each individual allowed us to mimic physical and psychological interactions among the people and the environment more realistically. Similarly, consideration of extended Decision Field Theory allowed us to represent human's decision deliberation process. In addition, consideration of a rich set of attributes for the environment as well as people has allowed us to mimic a real shopping mall environment closely. A crowd simulation 


\section{$X i$, Lee and Son}

model constructed based on the proposed pedestrian model and data (survey and observations) has been used to conduct several experiments. Our experimental results revealed several interesting findings such as 1) consideration of human's vision into the SFM is found to be significant, 2) impact of the number of unplanned shoppers on the profit of the mall is more significant when the mall is more crowded , 3) the profit score significantly increases as the percentage of the group shoppers increases, and 4) the shopping mall gains a higher profit if similar-type shops are placed close to each other. Our future work will employ extended Decision Field Theory for shopping path planning according to the dynamically changing environment.

\section{REFERENCES}

Busemeyer, J. R., and A. Diederich. 2002. Survey of decision field theory. Mathematical Social Science 43: 345-370.

Busemeyer, J. R., and J. T. Townsend. 1993. Decision field theory: A dynamic-cognitive approach to decision making in an uncertain environment. Psychological Review 100: 432-459.

Gaskell, G. D., and R. J. Benewick. 1987. The Crowd in Contemporary Britain. Sage, London.

Helbing, D., L. Buzna, A. Johansson, and T. Werner. 2005. Self-organized pedestrian crowd dynamics: experiments, simulations, and design solutions. Transportation Science 39: 1-24.

Helbing, D., I. Farkas, and T. Vicsek. 2000. Simulating dynamical features of escape panic. Nature 407: 487-490.

Lee, S., Y. Son, and J. Jin. 2008. Decision field theory extensions for behavior modeling in dynamic environment using Bayesian belief network. Information Sciences 178: 2297-2314.

Moussaï, M., D. Helbing, S. Garnier, A. Johansson, M. Combe, and G. Theraulaz. 2009. Experimental study of the behavioural mechanisms underlying self-organization in human crowds. Proceedings of The Royal Society B 276: 2755-2762.

Parisi, D., M. Gilman, and H. Moldovan. 2009. A modification of the social force model can reproduce experimental data of pedestrian flows in normal conditions. Physica A 388: 3600-3608.

Xia, Y., S. C. Wong, and C. Shu. 2009. Dynamic continuum pedestrian flow model with memory effect. Physical Review 79(066113): 1-8.

Zacharias, J., T. Bernhardt, and L. Montigny. 2005. Computer-simulated pedestrian behavior in shopping environment. Journal of Urban Planning and Development 131(3): 195-200.

\section{AUTHOR BIOGRAPHIES}

HUI XI is a Ph.D. student in the Department of Systems and Industrial Engineering at U. of Arizona. She received her BS in Automation Measurement and Control from Harbin Institute of Technology in China. She is a student member of INFORMS. She can be reached at <huix@email. arizona.edu>.

SEUNGHO LEE is a researcher at Samsung Mechatronics \& Manufacturing Technology Center. He received his BEng in IE from Korea University in 1999, MS in IE from Texas A\&M in 2005, and PhD in SIE from $U$ of Arizona. His research focuses on application of distributed simulation and simulation of human decision-making. He can be reached at<mountlee@gmail.com>.

YOUNG-JUN SON is a Professor of Systems and Industrial Engineering at U. of Arizona. He is an associate editor of the Int. Journal of Modeling and Simulation and the Int. Journal of Simulation and Process Modeling. He has received the SME 2004 Outstanding Young Manufacturing Engineer Award, the IIE 2005 Outstanding Young Industrial Engineer Award, IERC Best Paper Awards (2005 in modeling and simulation; 2008 in homeland security), and the Best Paper of the Year Award in 2007 from Int. Journal of Industrial Engineering. He can be reached by email at <sonesie.arizona.edu> 